Microcracking Study of Carbon Fiber Composite Materials for Starshade Petals

R. E. Rudd, A. van Buuren, J. N. Florando, H. A. Ishii, E. A. Withey, P. J. Wozniakiewicz, K. Fisher, B. A. Macintosh

June 4, 2012 
This document was prepared as an account of work sponsored by an agency of the United States government. Neither the United States government nor Lawrence Livermore National Security, LLC, nor any of their employees makes any warranty, expressed or implied, or assumes any legal liability or responsibility for the accuracy, completeness, or usefulness of any information, apparatus, product, or process disclosed, or represents that its use would not infringe privately owned rights. Reference herein to any specific commercial product, process, or service by trade name, trademark, manufacturer, or otherwise does not necessarily constitute or imply its endorsement, recommendation, or favoring by the United States government or Lawrence Livermore National Security, LLC. The views and opinions of authors expressed herein do not necessarily state or reflect those of the United States government or Lawrence Livermore National Security, LLC, and shall not be used for advertising or product endorsement purposes.

This work performed under the auspices of the U.S. Department of Energy by Lawrence Livermore National Laboratory under Contract DE-AC52-07NA27344. 


\title{
Microcracking Study of Carbon Fiber Composite Materials for Starshade Petals
}

\author{
Robert E. Rudd, Anthony van Buuren, Karl Fisher, Jeffrey N. Florando, Hope A. Ishii, \\ Elizabeth A. Withey, Penelope J. Wozniakiewicz, and Bruce A. Macintosh (PI) \\ Lawrence Livermore National Laboratory
}

May, 2012

\begin{abstract}
We have conducted materials tests of representative carbon composite materials for starshade petals. The principal goal of these tests is to assess whether the level of microcracking of the materials as the petals are stowed or deployed (or even as they are handled during assembly) is likely to cause a problem with meeting the tight dimensional tolerances required for starlight suppression. Cracking can cause unexpected deformation, and the cracks can act as reservoirs for gases. It may also lead to roughening of the optical edge whose sharpness minimizes undesirable scattering of light. We use mechanical tests, microscopy, acoustic tomography and small-angle $\mathrm{x}$-ray scattering to characterize the microcracking. These tests have found no large cracks (>100 microns) at bending strains up to 0.005 , the strain at which audible popping occurred. The stress-strain curves did not display any anomalies until a precipitous drop in stress at a strain of $\sim 0.012$ due to the formation of a transverse crack. Analysis of SAXS data is on-going which will quantify microcrack distributions at the sub-micron level. We find no large cracks (>100 microns) at the design strain tolerance for non-critical structural elements (0.005); analysis is on-going to quantify nanoscale cracking at the strains of critical structural elements (0.0005).
\end{abstract}

\section{Overview}

As materials are put under a load, they may undergo deformation that is irreversible; e.g. a bend that does not completely straighten when the load is removed. For composite materials that irreversible deformation may be due to cracking or creep. Creep results from the flow of defects in the material over a long period of time in response to the stresses in the material. Cracking may occur due to tension, such as at the outer radius of a bent longeron (mode 1 fracture). It may also occur due to shear (mode 2 fracture). In composites, fracture often occurs at the interface of the fiber and the matrix. The fibers are quite strong compared to the interface, and mode 1 or mode 2 fracture can occur at the interface. In compression, fiber composites suffer from a buckling failure in which the fibers buckle and the fiber-matrix interface fails. A large crack may run entirely across a structural element, causing failure of that element. Even without complete failure, cracks at the microscopic level can cause deformation of the material and they can be reservoirs for absorbed gases. Since the efficacy of the occulter requires tight dimensional control, it is a concern to have even small levels of irreversible deformation or deformation that relaxes slowly as the material out-gases.

Bending of fiber composites is often accompanied with a popping sound due to micro-fracture events. Small cracks form at the fiber-matrix interface during deformation producing this sound. The tough matrix may prevent these cracks from running across the structural member and causing 
complete failure, but the micro-cracks accumulate under continued fracture. Experiments in the literature have characterized carbon-carbon composite micro-cracking using the acoustic emission method [Bussiba 2008a, 2008b]. The material they studied is similar to, but not identical to, the kind of composite best suited to the starshade petals. They placed a sample in a rig for flexural and bending tests and recorded acoustic emission events as the sample was deformed. They found that fracture began at about $0.1 \%$ strain (1000 $\mu$ strain). Siron et al. [Siron 1999] have also used acoustic emission to study fracture processes in carbon composites. For comparison, the design specs for the starshade petals are that no component is subjected to more than a $0.5 \%$ strain and the battens are subjected to $<0.05 \%$ strain in stowing, deployment or operation.

We have conducted experiments to characterize micro-cracking in starshade petal composite materials subjected to strains representative of petal stowage and deployment. In particular, we have conducted 4-point bending tests and used optical microscopy, acoustic tomography, small angle x-ray scattering (SAXS) to determine the extent and character of micro-cracking in the composite samples. The tomography has been done both prior to, and following, deformation in a 4-point bending rig. The SAXS measurements have been performed on a series of samples deformed to different strains from asreceived to beyond the threshold for audible cracking. The goal is to determine the initial crack/void density (or put an upper bound on it) and the threshold strain for further micro-cracking.

\section{Sample Specifications}

We have tested 15 straight cut test samples $12.7 \mathrm{~mm}$ wide $\times 50.4 \mathrm{~mm}$ long $\times 0.48 \mathrm{~mm}$ thick fabricated by Patz Materials \& Technology specifically for this TDEM project. The material consists of layers of M55J high-modulus carbon fibers embedded in resin rotating by $60^{\circ}$ from layer to layer. Both surfaces have the fibers oriented transverse longitudinal axis (which we indicate as $0^{\circ}$ ). These samples are representative of the kind of composite materials that will be used for the optical edge of the starshade, with an opportunity for further optimization of the materials going forward in order to obtain slight improvements for the CTE and mechanical properties. The carbon composite is also similar in some respects to what would be used for the structural elements (battens and longerons), but the layup tested here is different than that of the pultruded structural rods.

\section{Bending Tests}

Bending tests have been performed in order to subject the samples to a specified level of strain in bending for subsequent characterization of the extent of microcracking. The bending tests also provide a stress-strain curve for the materials. These 4-point bend tests were conducted on an Instron 5900 (an electromechanical screw driven loading machine) with a $2 \mathrm{kN}$ load cell, guided by the ASTM standard D6272 [ASTM 2010]. The support span was $30 \mathrm{~mm}$ and the load span $10 \mathrm{~mm}$. The bending tests were run at a displacement rate of $3.48 \mathrm{~mm} / \mathrm{min}$. The displacement of the sample was measured using a laser extensometer model LE-05 which has a range of $10 \mathrm{~mm}$ full scale. A more detailed description of the method used for bending tests is given in Ref. [ASTM 2010].

The stress-strain curves obtained in the 4-point bend tests are shown in Figure 1. The initial part

of each curve at small strains represents elastic deformation. For sufficiently large strains, microcracking 
occurs. At a strain of 0.005 audible popping sounds were detected that were attributed to microcracking. At a strain of $0.012-0.013$ a large transverse crack formed causing the precipitous drop in stress apparent on curves $A$ and $G$ in Figure 1. Sample A was taking to complete failure at a strain of 0.022. This failure strain agrees with the value reported by Baral et al. for another M55J composite to within the error bars [Baral 2008].

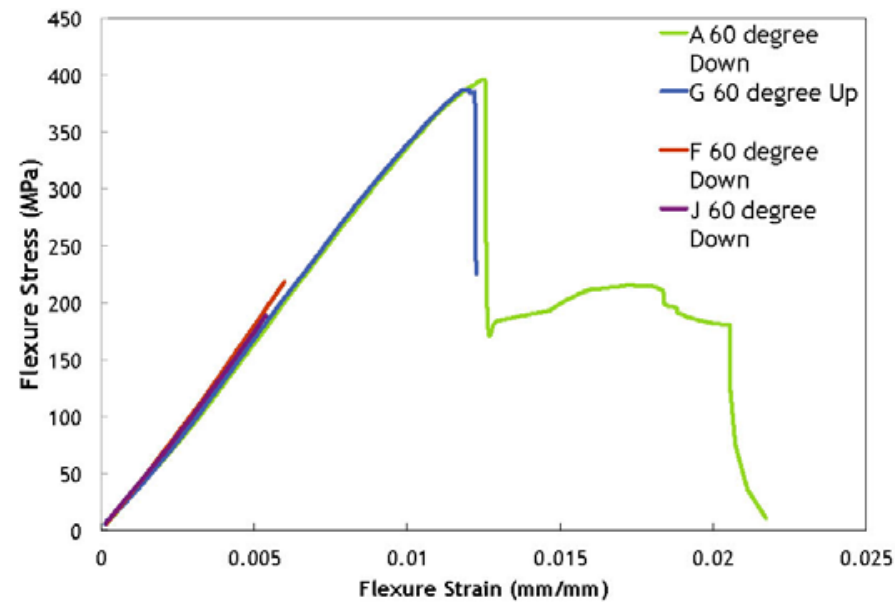

Figure 1. Stress-strain curves measured in the 4-point bend tests. The stress-strain curves from 4 samples are overlaid for comparison. The samples are nominally identical, apart from sample $\mathbf{G}$ being arranged in the bend test differently, with the surface with the 60 degree texture up rather than down. The notation 60 degree up or down refers to the orientation texture pattern on the specimen surface with respect to the transverse direction. The fibers are perpendicular to the long direction of the specimen on both of the outer surfaces. The samples were strained to different levels and then unloaded for further analysis to determine the microcracking at those strain levels. Sample A was strained to fail completely due to multi-layer fracture.

The stress-strain curves for the 7 SAXS samples are shown in Figure 2.

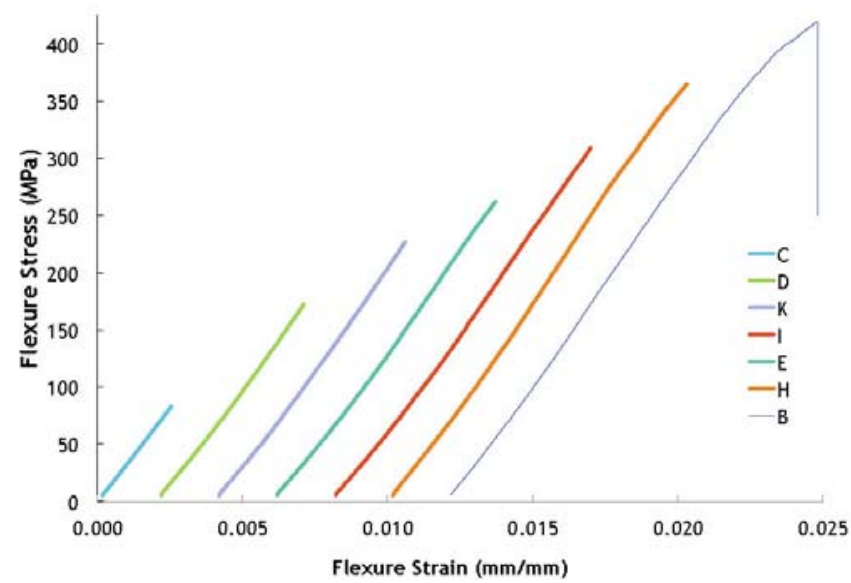

Figure 2. Stress-strain curves measured in the 4-point bend tests: the 7 samples for SAXS measurements (offset horizontally to separate the curves). All samples were loaded with the same orientation. The samples were strained to different levels and then unloaded for further analysis to determine the microcracking at those strain levels. 


\section{Optical Microscopy}

Optical micrographs of the carbon fiber composite have been taken prior to, and after, bend testing. Most of the samples showed no evidence of damage due to the bending tests. Samples $G$ and $B$ exhibited a transverse surface crack that spanned the width of the sample but did not cross through the thickness. These samples showed a drop in stress at a strain of $\sim 0.012$, as shown in Figure 1 and Figure 2 , respectively. An optical micrograph of the fracture is shown in Figure 3. The image also shows the rough surface of the sample in the left panel. This roughness was present in the as-received sample and is the result of the fabrication process. The opposite surface is smooth, as shown in the right panel.
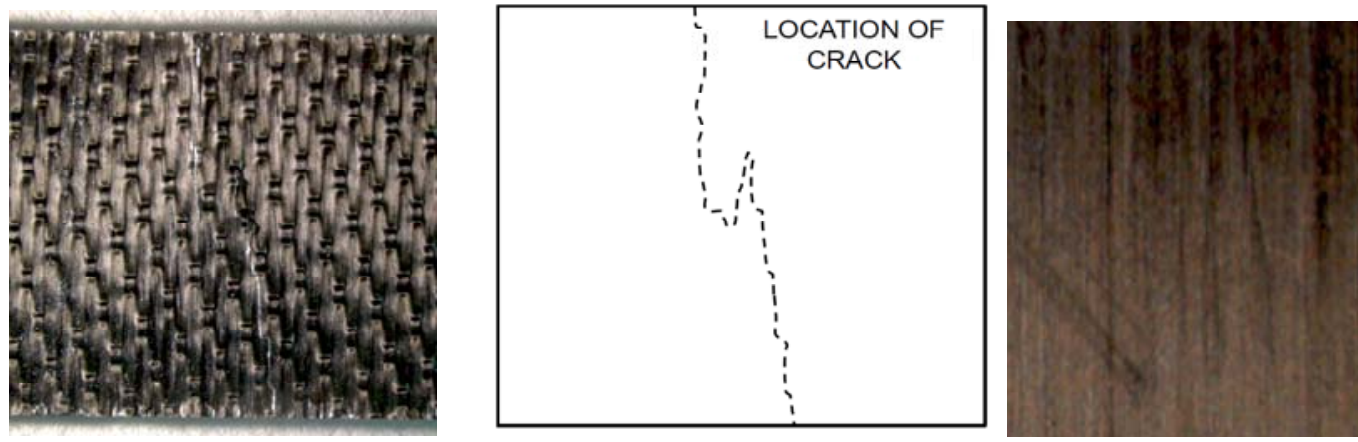

Figure 3. (left panel) Optical micrograph of a sample of the carbon fiber composite sample G, subjected to a bending strain of $\sim 012$ causing fracture of the surface fiber layer. The stress-strain curve for this sample is shown in Figure 1. The fracture did not propagate into the lower layers of the composite. The entire 12.7 $\mathrm{mm}$ width of the sample is visible top to bottom. (center panel) A schematic illustration of the morphology of the crack. (right panel) The opposite surface of the samples is smooth.

Sample A was subjected to a strain of 0.022 and failed completely. The rough edge of the resulting fracture is shown in Figure 4. The different layers of the composite fractured at different locations and with different morphologies, as shown in Figure 5, 6 and 7.

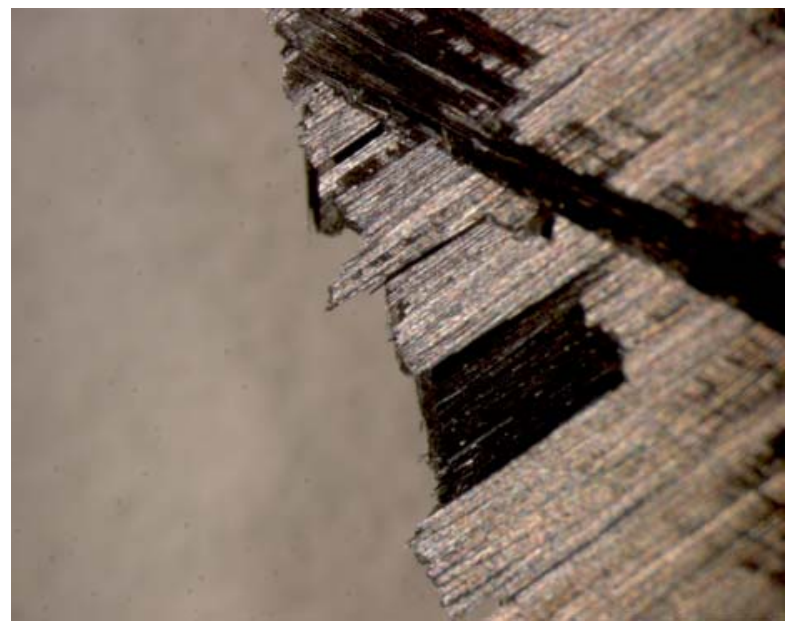

Figure 4. Optical micrographs of the carbon fiber composite materials. Fractured edge of a sample taken to complete failure (the green curve of Figure 1a). A rough fracture is visible, exposing different layers of the composite. 


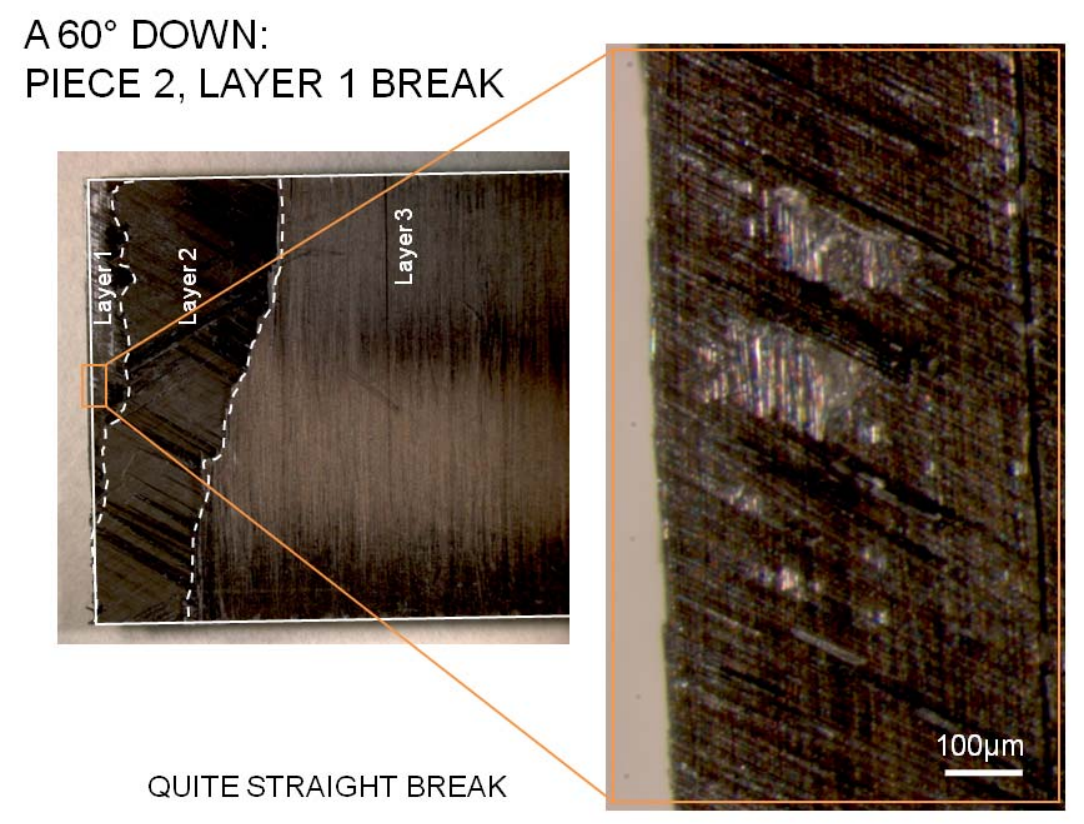

Figure 5. A close-up view of the fracture surface at the rough side of sample A. Layers 1, 2 and 3 indicate the rough face, interior and smooth face, respectively.

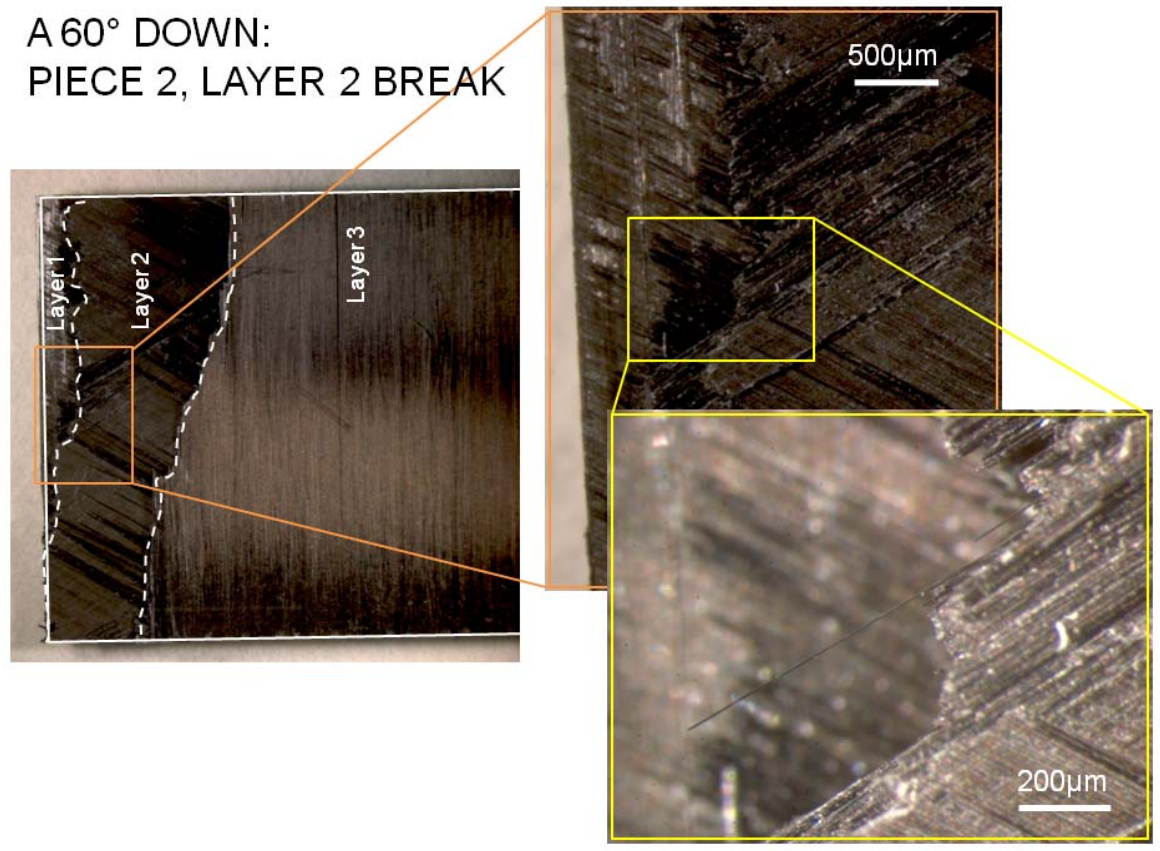

Figure 6. A close-up view of the fracture surface at the interior of sample A. The different layers of the composite are visible. 


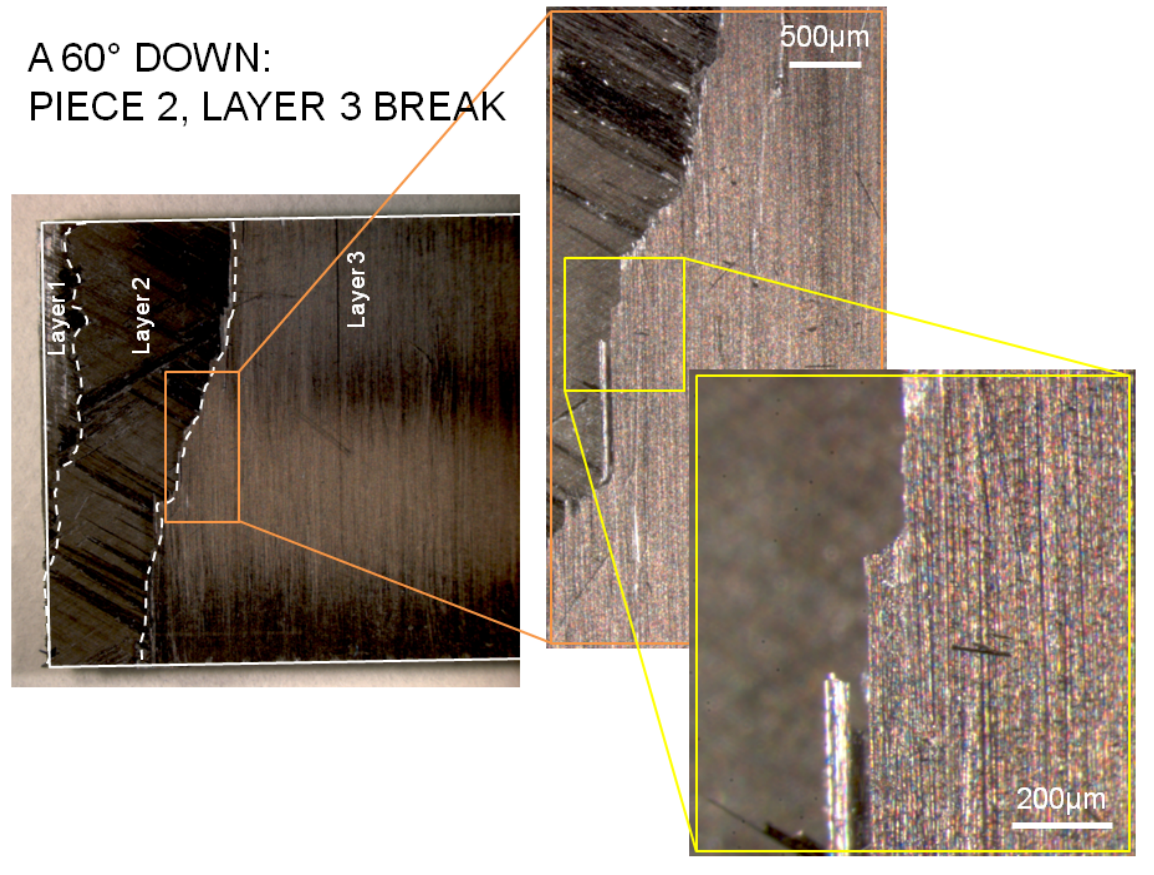

Figure 7. A close-up view of the fracture surface at the smooth surface of sample A. The transverse orientation of the outer fibers is visible.

\section{Acoustic Tomography}

Acoustic tomography tests were performed in order to characterize relatively large cracks by imaging (cracks $>100$ microns) and by the total level of acoustic attenuation (cracks $>1$ micron). The acoustic tomography was performed on a sample subjected to a strain of 0.005 , the level of strain at which audible popping but no visible crack occurred in the 4-point bend test. Acoustic tomography is a kind of non-destructive testing. In these tests, acoustic waves are run through a sample in order to detect and map inhomogeneities within the sample. The samples are immersed in a fluid that provides acoustic coupling to the sample. We have used water. A high frequency transducer produces acoustic waves that propagate through the fluid, into the sample where there is some loss due to absorption or scatter, back into the fluid and are then detected by another transducer in the fluid. Any variations in the acoustic impedance of the material induce scattering and/or absorption of the waves, and the attenuation of the acoustic signal can be used to construct a map of the heterogeneities in the material including cracks and voids. If the material has many small cracks that are too small to image, it is possible to infer the extent of microcracking from the level of acoustic attenuation. Due to the wavelength of the acoustic waves, the technique can only image cracks and other inhomogeneities down to about 100 microns in size. The technique is sensitive to the overall population of cracks larger than about 1 micron through the total attenuation of the signal. It provides a valuable test of the integrity of the as-receive materials and whether large cracks form as a result of the bending tests. A review of the acoustic tomography technique is given in Ref. [Birks 1991]. 
The parts were acoustically scanned using an industrial pulse-echo immersion scanner (Panametrics). The scan recorded the point to point transmission amplitude loss through the thickness of the composite coupon. The color differences indicate attenuation of the acoustic signal through the material thickness due to material differences. The $15 \mathrm{MHz}$ transducer was chosen after several attempts to reveal the layup pattern. This will not image individual fibers, (too small) just larger features on the order of 100um.

Results of the acoustic tomography tests are shown in Figure 8. No microcracking was observed at strains up through the threshold for audible cracking at a strain of 0.005 . The pattern observed in these images is due to acoustic scattering from surface roughness. These findings exclude the formation of large cracks $>100$ microns in size.

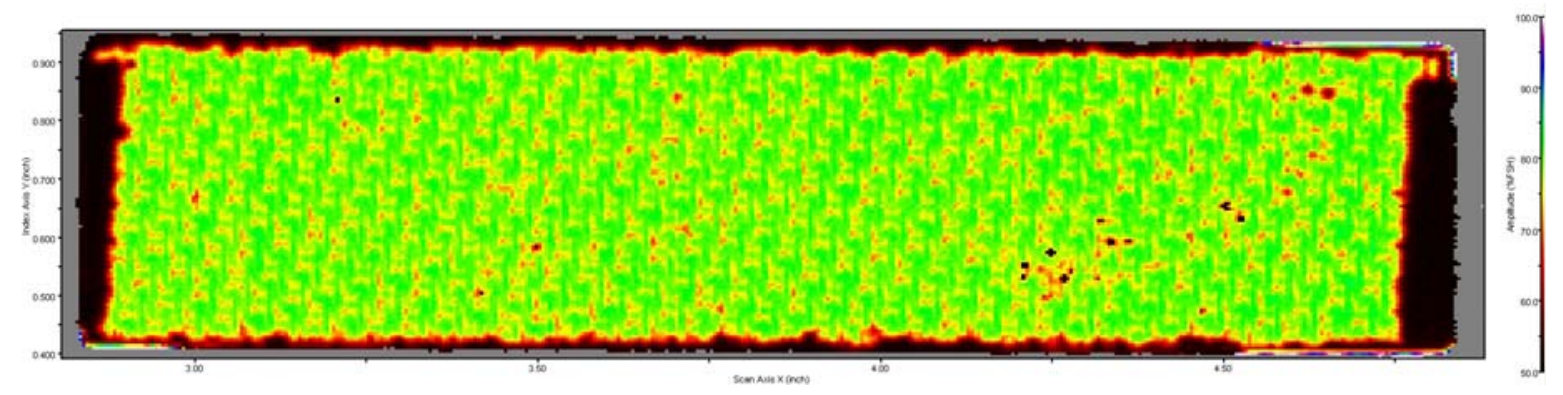

Figure 8. Acoustic tomography map of the fiber composite materials. The coloring corresponds to the amplitude of the acoustic signal, as indicated in the color bar to the right. No microcracking was observed at strains up through the threshold for audible cracking at a strain of $\mathbf{0 . 0 0 5}$. The pattern observed in these images is due to acoustic scattering from surface roughness. The dark areas at the edges of the specimen are where it was supported in the tank.

\section{Small-Angle X-ray Scattering (SAXS)}

SAXS measurements use $x$-ray scattering to detect inhomogeneities in a sample. A bright $x$-ray source generates monochromatic $x$-rays that scatter off variations of the electron density in a sample, including the drop of the electron density in a crack or void. The scattered $x$-rays are recorded on a 2dimensional flat $\mathrm{X}$-ray detector situated behind the sample with the plane of the detector perpendicular to the direction of the incident $x$-ray beam. The scattering pattern is analyzed to determine information on the structure of the sample. The SAXS technique is sensitive to cracks from a few nanometers up to a $\sim 100 \mathrm{~nm}$. Another technique, Ultra-Small-Angle X-ray Scattering (USAXS), is sensitive to larger cracks up to a micron. In either technique, the beam can be positioned at different points in the sample in order to determine spatial variation in the crack size distribution. A monograph is available that describes the SAXS technique in detail [Glatter and Kratky 1982].

We have used the Advanced Light Source (ALS), Beamline 7.3.3, at the Lawrence Berkeley National Laboratory to obtain SAXS data for the starshade carbon composite material. Figure 9 shows the scattering pattern. The 6 -fold symmetric scattering pattern is due to the $\left(60^{\circ}, 0^{\circ},-60^{\circ}\right)$ layup of the composite. The individual fibers are 7 microns in diameter, so they are too large to affect the SAXS signal. The six-fold symmetry is a result of anisotropy of microcracks and smaller features along the interface of the fibers and the resin matrix. 


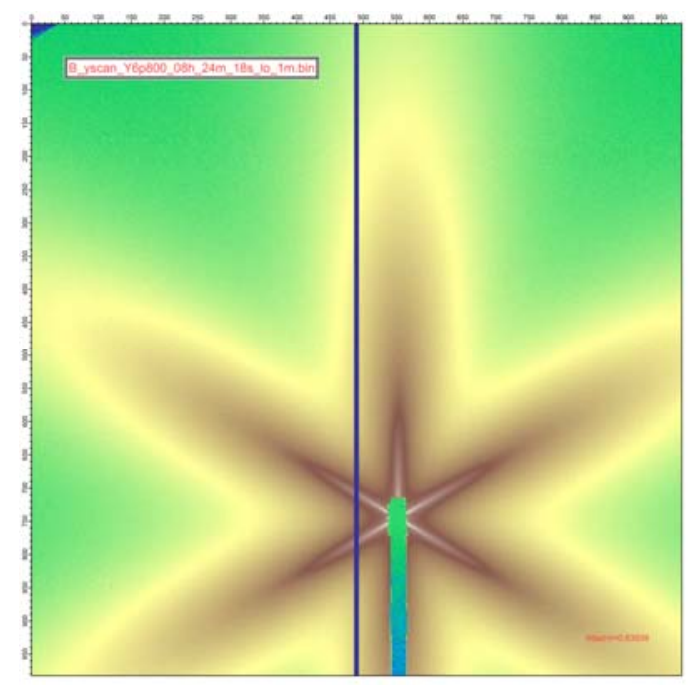

Figure 9. Small-angle x-ray scattering (SAXS) plot for the carbon composite sample B, the sample subjected to the largest strain of the 7 SAXS samples. The 6-fold symmetric scattering pattern is due to the $\left(60^{\circ}, 0^{\circ},-60^{\circ}\right)$ layup of the composite. Sections through the scattering pattern were used to determine the microcrack distribution.

In order to relate the SAXS data of Figure 9 to a microcrack size distribution a quantitative analysis is needed. Standard SAXS analysis packages assume that the defects causing the scattering are isotropic and homogeneous throughout the region expose to the $x$-ray beam. The six-fold scattering pattern shown in Figure 9 is clearly not isotropic, so a generalized analysis is needed. The approach is to consider the SAXS intensity at a specified angle chosen to be sensitive to the dimension of the microcracks running along the fiber-matrix interface.
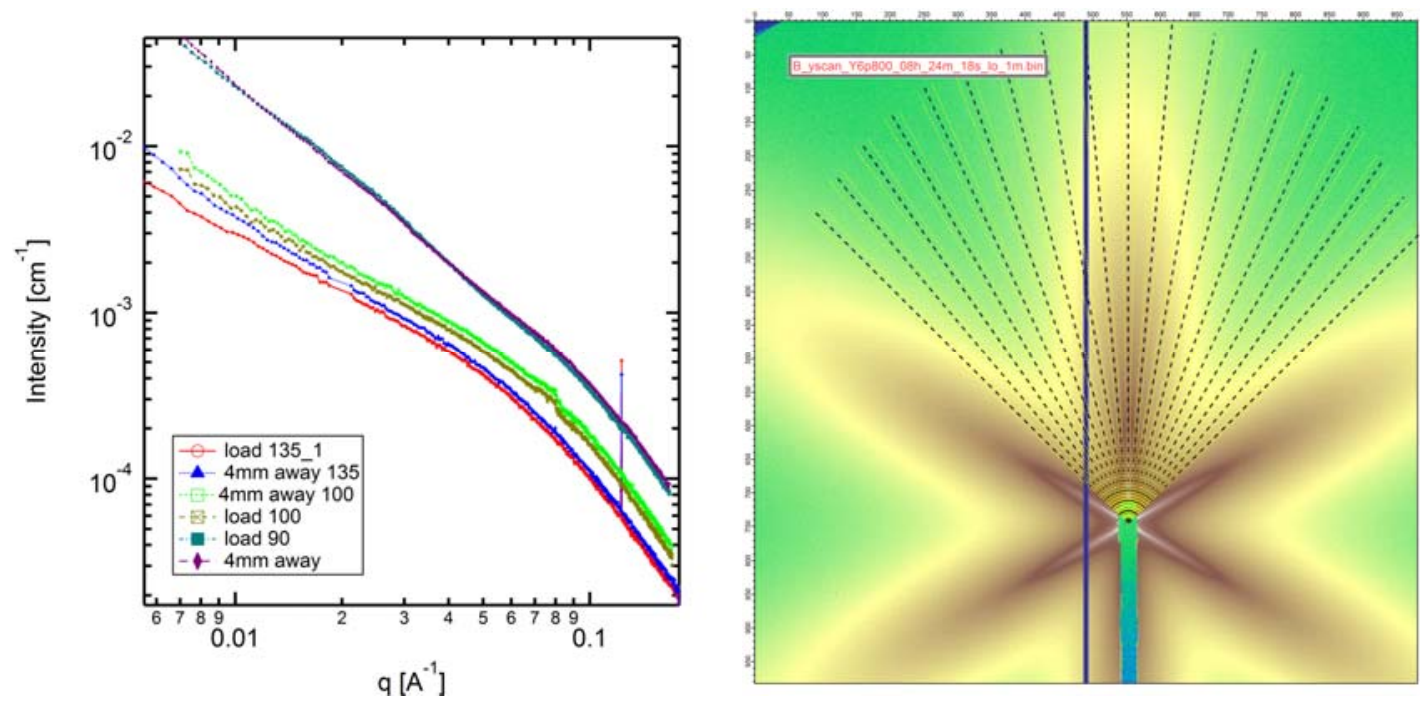

Figure 10. Lineout from the SAXS scattering intensity (Figure 9). 
Several lineouts through the scattering data are shown in Figure 10. These lineouts correspond to two positions on sample $B$, one at the center of the loading span and one at $4 \mathrm{~mm}$ from the center. The scattering intensity was summed as a function of the scattering wavevector $q$ at the angle indicated $\pm 1^{\circ}$. The two highest intensity curves correspond to the scattering data through the center of the lobe at the two positions on the sample. With this reduction of the data, the analysis can proceed using standard techniques. In general, the goal is to relate the scattering intensity to the properties of the sample, in this case the cracks. For example, in the Guinier regime the scattering intensity can be related to the radius of gyration of the scatterers according to [Putnam 2007]

$$
I(q)=I(0) \exp \left(-q^{2} R_{G}^{2} / 3\right)+C
$$

The SAXS analysis is on-going. The goal of this work is to characterize the crack size distribution in the sub-micron range, looking for thresholds in the behavior as the strain increases.

\section{Transmission Electron Microscopy (TEM)}

We have set aside some samples for future testing of the level, location, and morphology of microcracking using TEM characterization. TEM requires sample preparation with the focused ion beam (FIB) in order to obtain samples of a suitable size and surface condition. A small sample is cut from the larger sample used in the mechanical tests and tomography. We have potted a sample in epoxy and stored it for future FIB and TEM work.

\section{Conclusion}

We have conducted material tests to characterize microcracking due to bending, such as might be experienced by the carbon composite materials in the starshade petals during stowing, deployment or even handling during assembly. These tests have found no large cracks ( $>100$ microns) at bending strains up to 0.005 , the strain at which audible popping occurred. The stress-strain curves did not display any anomalies until a precipitous drop in stress at a strain of $\sim 0.012$ due to the formation of a transverse crack. Analysis of SAXS data is on-going which will quantify microcrack distributions at the sub-micron level.

An interpretation of these results is that failure occurs at several levels within the carbon fiber composites. At very low levels of strain, the material bends elastically. At a strain of $\sim 0.1 \%$ microcracking begins to create a low density of extremely small cracks. This inference is based on the work of Bussiba et al. [Bussiba 2008a], since we have not observed these cracks in our experiments. At a strain of $\sim 0.5 \%$ microcracks are sufficiently large that audible popping occurs. These cracks are less than 100 microns in size, and have no pronounced effect on the stress-strain curves. Then at a strain of $\sim 1.2 \%$ a large transverse crack forms that fractures one layer of the composite leading to a large drop in the stress, but not leading to complete failure of the material. Finally, at a strain of $\sim 0.022$ the material fails completely due to fracture of all of the layers of the composite.

According to this interpretation, critical structural elements such as the battens which experience strains $<0.05 \%$ in stowing, deployment or operation [Kasdin 2011] would bend elastically and microcracking would not take place that could affect their performance. Provided they are handled 
carefully during assembly, microcracking is not an issue for them. The optical edge also experiences small strains during stowing, deployment or operation of $<0.1 \%$ because it is only $\sim 0.5 \mathrm{~mm}$ thick. The longerons experience larger strains $\sim 0.5 \%$, near the threshold for audible popping. Microcracking does occur at this level of strain, but the length of the longerons is not so critical for maintaining the critical dimensions of the petals. Even strains of $\sim 0.5 \%$ are well below the threshold for failure.

Cracking may also be a concern in other areas of the starshade. We have not assessed whether cracking might happen on the sharp edge of the optical edge material. In this case, the concern is that cracking might roughen the surface due to the cracks themselves or the exposure of fibers and this roughness could cause additional light scattering. So far it appears that the material is sufficiently robust, and there is nothing to indicate that edge cracking will be a problem; we make note of it here as something related to the microcracking tests but not explicitly measured.

\section{Acknowledgments}

We thank Jeremy Kasdin, Douglas Lisman, Robert Huber, Dmitry Savransky, and Lenny Summers for useful discussions. We thank Douglas Lisman for providing the composite samples. This work was performed under the auspices of the U.S. Department of Energy by Lawrence Livermore National Laboratory under Contract DE-AC52-07NA27344. LLNL-TR-559615.

\section{References}

[ASTM 2010] ASTM D6272-10. Standard test method for flexural properties of unreinforced and reinforced plastics and electrical insulating materials by four-point bending (ASTM Intl., West Conshohocken, PA, 2010).

[Baral 2008] N. Baral, H. Guezenoc, P. Davies and C. Baley, "High modulus carbon fibre composites: Correlation between transverse tensile and mode I interlaminar fracture properties," Mater. Lett. 62, 1096-1099 (2008). http://dx.doi.org/10.1016/j.matlet.2007.07.071

[Birks 1991] A.S. Birks, R.E. Green, and P. Mclntire, eds. Ultrasonic Testing. The Nondestructive Testing Handbook, second edition. Vol.7. (American Society for Nondestructive Testing, 1991).

[Bussiba 2008a] A. Bussiba, M. Kupiec, R. Piat, and T. Böhlke, Carbon 46, 618 (2008);

http://dx.doi.org/10.1016/j.carbon.2008.01.020

[Bussiba 2008b] A. Bussiba, M. Kupiec, S. Ifergane, R. Piat, and T. Böhlke, Composites Science and Technology 68, 1144-1155 (2008). http://dx.doi.org/10.1016/j.compscitech.2007.08.032

[Glatter and Kratky 1982] O. Glatter and O. Kratky, Small Angle X-ray Scattering (Academic Press, 1982).

[Kasdin 2011] N. J. Kasdin, D. N. Spergel, R. J. Vanderbei, D. Lisman, S. Shaklan, M. Thomson, P. Walkemeyer, V. Bach, E. Oakes, E. Cady, S. Martin, L. Marchen, B. Macintosh, R. E. Rudd, J. Mikula, D. 
Lynch, “Advancing Technology for Starlight Suppression via an External Occulter," Proc. SPIE 8151, 81510J (2011). http://link.aip.org/link/doi/10.1117/12.894497

[Putnam 2007] C. D. Putnam, M. Hammel, G. L Hura, and J. A. Tainer, Quarterly Reviews of Biophysics 40, 191-285 (2007). http://dx.doi.org/10.1017/S0033583507004635

[Siron 1999] O. Siron, G. Chollon, H. Tsuda, H. Yamauchi, K. Maeda, and K. Kosaka, Carbon 38, 1369-13 (2000). http://dx.doi.org/10.1016/S0008-6223(99)00270-5 